\title{
BMJ Open Medical students' opportunities to participate and learn from activities at an internal medicine ward: an ethnographic study
}

\author{
A Hägg-Martinell, ${ }^{1} \mathrm{H}$ Hult, ${ }^{2} \mathrm{P}$ Henriksson, ${ }^{1}$ A Kiessling ${ }^{1}$
}

To cite: Hägg-Martinell $A$, Hult $\mathrm{H}$, Henriksson $\mathrm{P}$, et al. Medical students' opportunities to participate and learn from activities at an internal medicine ward: an ethnographic study. BMJ Open 2017;7:e013046. doi:10.1136/bmjopen-2016013046

- Prepublication history for this paper is available online. To view these files please visit the journal online (http://dx.doi.org/10.1136/ bmjopen-2016-013046).

Received 16 June 2016 Revised 20 November 2016 Accepted 5 January 2017

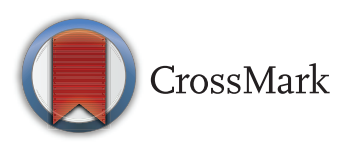

${ }^{1}$ Department of Clinical Sciences, Danderyd Hospital, Karolinska Institutet, Stockholm, Sweden ${ }^{2}$ Department of Clinical Science, Intervention and Technology, Karolinska Institutet, Stockholm, Sweden

Correspondence to A Hägg-Martinell; ann.hagg-martinell@ki.se

\section{ABSTRACT}

Objectives: To optimise medical students' early clerkship is a complex task since it is conducted in a context primarily organised to take care of patients. Previous studies have explored medical students' perceptions of facilitation and hindrance of learning. However, the opportunities for medical student to learn within the culture of acute medicine care have not been fully investigated. This study aimed to explore how medical students approach, interact and socialise in an acute internal medicine ward context, and how spaces for learning are created and used in such a culture.

Design and setting: Ethnographic observations were performed of medical students' interactions and learning during early clerkship at an acute internal medicine care ward. Field notes were taken, transcribed and analysed qualitatively. Data analysis was guided by Wenger's theory of communities of practice.

Participants: 21 medical students and 30 supervisors participated.

Results: Two themes were identified: Nervousness and curiosity — students acted nervously and stressed, especially when they could not answer questions. Over time curiosity could evolve. Unexplored opportunities to support students in developing competence to judge and approach more complex patient-related problems were identified. Invited and involved - students were exposed to a huge variation of opportunities to learn, and to interact and to be involved. Short placements seemed to disrupt the learning process. If and how students became involved also depended on supervisors' activities and students' initiatives.

Conclusions: This study shed light on how an acute internal medicine ward culture can facilitate medical students' possibilities to participate and learn. Medical students' learning situations were characterised by questions and answers rather than challenging dialogues related to the complexity of presented patient cases. Further, students experienced continuous transfers between learning situations where the potential to be involved differed in a wide variety of ways.

\section{INTRODUCTION}

Medical students develop professionally mainly through the experiences that they

\section{Strengths and limitations of this study}

- Qualitative studies are performed in a defined context and thereby create an opportunity for a deeper analysis of the studied context.

- The ethnographic design provided a potential to gain a deeper understanding of participants' actions and interactions, and the extended observation period of a large number of staff and students increased the findings' trustworthiness.

- A limitation of ethnographic studies is the risk of researcher bias, as the researcher is used as a tool during the observations. A researcher with preunderstanding and experience of the studied context may not recognise important aspects. On the other hand, an experience of the studied context might allow the analysis to be performed in more depth.

- The quality of ethnographic data depends on whether participants are willing to participate and if they act as they would have done if not observed. Initially, some staff were a bit cautious, but all agreed to participate and soon expressed that the research was important and that they wanted to contribute.

- Investigator triangulation was applied and all researchers were engaged in the analysis, which meant that different professional perspectives and experiences could be applied in order to ensure credibility.

acquire during clinical work. ${ }^{1}$ The challenge of clinical learning is to facilitate development from novice to practising physician. ${ }^{2}$ Students need time to organise medical knowledge into relevant clinical competence, and clerkship learning should thus start early. ${ }^{3}$ However, early clerkship is a complex task since learning takes place in a context that is primarily organised to effectively take care of patients. ${ }^{4}$

Medical students' professional learning takes place in a healthcare context based on interaction and mutual learning between 
individuals and groups. We have therefore based this study on social theory. Vygotsky's theory of learning takes the sociological aspects of learning in the social context into account. The interactions and interpersonal communications allow an individual to integrate the experience of others into his or her own learning. The cognitive development is dependent on a fusion of concrete experience of context and of systematic, abstract and theoretical knowledge. ${ }^{5}$

Wenger has described professional team collaboration and the commitment they conduct together as a community of practice. ${ }^{6}$ A community of practice can be described in three dimensions: a joint enterprise means that the members contribute to the community; mutual engagement is when its members establish norms and relationships and a shared repertoire is communal resources, like shared norms for collaboration and problem solving, language, tools and routines. Learning is a central and important aspect of a community of practice. It can be characterised by shared histories of learning, mutual engagement in pursuing an initiative together and sharing of some significant learning. The team members discover how to engage and what helps and what hinders their learning. In a professional community, the members share goals, values and methods. The healthcare staff and the care they perform is an example of a community of practice. All members of a community are expected to contribute to learning and to development of the community. Even a novice such as a student can contribute to and be accepted as a member of a community if the members allow him or her to do so. However, according to Wenger, students' participation in a community can be legitimate but is often peripheral. ${ }^{6}$ During a stay at a ward, a student should enter the community and pass through its boundary. Boundaries between communities can create separation and be a basis for exclusion, fragmentation and disconnection, but can also create areas of extraordinary learning, places where different perspectives meet and where new opportunities arise. When individuals enter a new community, they bring competence and experience from other communities. In interaction with the new community they will contrast own experience with foreign competence. According to Wenger, the optimal condition for learning is a tension between previous experience and foreign competence. However, if an individual already has the competence needed to participate in the new community, or if the distance between previous experience and the foreign competence required is too wide, achievement of learning could be futile. ${ }^{7}$ The community of practice theory has developed over time and Wenger-Trayner and Wenger-Trayner ${ }^{8}$ have rephrased it as the theory of 'Learning in Landscapes of practice', where they highlight the importance of knowledgeability and of building a professional identity by travelling in a landscape of different and complex practices rather than by participating in one community of practice. Engagement, imagination and alignment are important aspects in this theory of social learning. Engagement characterises participation in the communities we belong to, and helps us to explore boundaries between different communities of practice we engage with during the journey. Imagination is when community members make assumptions about each other, recall the past and talk about their future. Alignment is a central aspect of a community's local regime of competence, but is also essential to the functioning of broader systems connecting communities to each other.

There are several interview, diary and observational studies conducted that explore if and how medical students learn in interactions and dialogues with patients and staff in healthcare workplaces. ${ }^{9} 10$

The qualities of the supervisor have been emphasised as fundamental for students' learning in workplaces. An excellent supervisor acts like a role model, focuses on teaching, creates safe learning situations, provides experiential learning opportunities, gives feedback, sets demands and stimulates learning. ${ }^{11}$ Also, competent and enthusiastic supervisors give students opportunities to interact with patients and allow them to take own responsibility. ${ }^{12}$ In the healthcare environment, students have a potential to form own professional identities. ${ }^{13}$ Furthermore, learning objectives, partnership with supervisors and continuity are fundamental aspects of a stimulating learning environment. ${ }^{14}$

Workplace learning in hospital settings has the potential to contribute to a socialisation process of students into their future profession. ${ }^{15}$ However, according to Atherley $e t a l^{16}$ learning and socialisation during workplace learning in internal medicine could be challenging. Many students experienced difficulty developing relationships with team members. Students with a positive attitude experienced a smoother transition. Many students were uncertain of their roles, concerned about the workload and desired guidance to meet clerkship demands. This transition resulted in varied outcomes from enjoyment, increased confidence and student development to disinterest. In addition, Köhl-Hackert et $a l^{17}$ have stated that medical students are valued to have supportive supervisors and to be an integrated member of the team at the internal medicine ward. An ethnographic study by Seabrook $^{18}$ has stated that medical students expressed that they often felt that they were in the way, and that their individuality was not valued.

We have, in a previous ethnographic study, shown that medical and nursing students' task on arrival to an acute internal medicine ward was to understand and to adapt to its culture, and to try to become accepted. Furthermore, the culture shaped a pattern for, and created conditions that challenged students' adaptation and also created a space for learning. ${ }^{19}$

To sum up, several studies have explored medical students' perceptions of qualities that facilitate or hinder clerkship learning in hospital settings. Previous studies 
on medical students' learning in the stressed acute internal medicine care contexts indicate that socialisation in such cultures can be especially challenging. These interesting findings need to be further explored. How do medical students approach, interact and socialise in an acute internal medicine ward context? How are spaces for learning created and used in such a culture? The aim of the present study was to explore how medical students approach, interact and socialise in an acute internal medicine ward culture, further to explore their opportunities to participate and to create space for learning in such a culture.

\section{METHOD}

\section{Study design}

An ethnographic study design was chosen to in depth explore the research questions. A wide data collection was performed during 2011-2013 observing workplace culture at an acute internal medicine care ward, including students' interaction and opportunities to learn within this context. Inspired by Hammersley and Atkinson, ${ }^{20}$ repeated observations and informal conversations took place during participation in the daily life on the ward for an extended period of time. The purpose was to watch what happened, listen to what was said and to ask questions that throw light on opportunities for medical students to learn in this community. In a previous study of this material, we have described the organisation, the physical environment and culture of this ward. ${ }^{19}$

\section{Study context}

During the study period, the assignment of the hospital was to perform acute healthcare for a population of $\sim 500000$ inhabitants and to perform clinical research and education. The observed ward belonged to the Department of Internal Medicine, one of the largest departments of the hospital. During the study period, 131 physicians worked at the department; 21 of them as senior consultants and 110 as resident physicians. The observed ward diagnosed and treated patients with various internal medicine diseases, mainly severe infections, pulmonary diseases, venous thromboembolism, intoxications, convulsive disorders and hypersensitive reactions.

The Swedish undergraduate medical education programme consists of 11 semesters. The latter part is characterised by recurrent periods of clerkship, mostly hospital based and in primary and community-based settings. The medical students in the ward were in the beginning of their clerkship training, that is, at semester five or six, and were scheduled to stay in the ward for $1-5$ days.

The intended learning outcomes of the course that includes clerkship at this ward are grouped into three categories: medical knowledge and understanding; skills and abilities; and judging and approaching.
Permission to perform the study in the ward was given by the head of the Department of Internal Medicine. Verbal informed consent was obtained from all concerned individuals in accordance with the Declaration of Helsinki after they had received information about the purpose and method of the study, and had been told that participation was voluntary. The medical students received oral information from their main supervisor and from the first author; senior consultants and residents received oral information from the first author. All medical students that performed workplace learning in the ward during the study period agreed to participate. Also, all supervisors and staff agreed to participate. All medical students, supervisors and staff were informed that the observer was a registered nurse but that her role at the ward was to conduct research and not to act as a nurse or as a supervisor. All patients and their relatives received information that education and research were performed at the hospital. More specific information about this study was given by the senior consultant in charge or by the first author.

\section{Data collection}

In total, 27 observations and 15 conversations were performed from early autumn 2011 to early spring 2013. Observations and informal conversations were performed in the ward, from Monday to Friday, between 8:00 and 16:30. A consecutive sampling of available observation days was applied, based on if students were present and if the observer had time to observe. Male and female students participated. Table 1 shows an overview of a working day in the ward.

Each observation session lasted on average 3-4 hours. The total duration of all observations and conversations were about 100 hours and included: 50 hours of interprofessional sit rounds, 25 hours of collaborative patientrelated paperwork at offices, 20 hours of patient care and consultation and 5 hours of informal patient-related

\begin{tabular}{ll} 
Table 1 & An overview of a working day in the ward \\
\hline Time & \\
\hline 8:00 & $\begin{array}{l}\text { Morning meeting } \\
\text { Interprofessional sit round } \\
\text { Examination of patients } \\
\end{array}$ \\
& $\begin{array}{l}\text { Consultations at other wards / departments } \\
\text { Paper work and patient-related duties }\end{array}$ \\
& $\begin{array}{l}\text { Coffee break } \\
\text { 10:00 }\end{array}$ \\
Paper work and patient-related duties \\
N1:00 & $\begin{array}{l}\text { Paper work and patient-related duties } \\
\text { Lunch }\end{array}$ \\
$14: 00$ & $\begin{array}{l}\text { Paper work and patient-related duties } \\
\text { Interprofessional sit round }\end{array}$ \\
$14: 30$ & $\begin{array}{l}\text { Paper work and patient-related duties } \\
\text { Paper work and patient-related duties }\end{array}$ \\
$16: 30$ & $\begin{array}{l}\text { Consultations at other wards / departments } \\
\text { Evening report }\end{array}$ \\
\hline
\end{tabular}


interactions, for example, in the corridor, in the staff lounge or in the treatment room. The conversations with participants were aimed to clarify participants' thoughts, to ask for explanations and to get a deeper understanding of the observations. The medical students were observed in interactions with supervisors, other staff members, patients, relatives or at individual learning sessions.

Twenty-one individual medical students and 30 supervisors (senior consultants and resident physicians, four nurses and one auxiliary nurse) were observed. In addition, patients and relatives could be observed when interacting with students. The medical students had short placements in this ward, but on the contrary several students were observed on several occasions. The last observations mostly gained repetitive information, thus saturation was assumed.

Field notes were taken during the observations and conversations and transcribed later the same day. ${ }^{20}$ Though the span of memory for field observations is short, it is important that the notes are written as soon after the event as possible. ${ }^{21}$ The first author performed all the observations and conversations. Her only relationship to the participants was as a researcher. She had no relationship to them as a nurse or a supervisor either before, during or after the observations. The observer wore hospital dress during the observations and conversations. All observations were gathered by a 'marginal participant' technique, in which the researcher plays only a minimal role in the social action they are observing. $^{20}$

\section{Data analysis}

Data analysis started during the fieldwork period. The analysis in this study was based on observations when medical students were present at the ward. An inductive approach was used. With the aim in mind, we used Wenger's ${ }^{6}$ three dimensions: a joint enterprise; a mutual engagement and a shared repertoire as a basis to find patterns when analysing the data. When the analysis revealed findings that needed to be observed in more detail, the data collection was modified. An example was that medical students left the ward during several occasions together with their supervisor to examine patients at other departments that needed their expertise. In the light of the revised theory of Community of Practice on learning by travelling between communities, ${ }^{8}$ we assumed that these 'expeditions' might contribute to learning and affect future interactions at the acute medical ward, though the data collection was expanded to include observations even of these learning activities. All transcriptions related to the aim were included in the analysis. The analysis of the data involved six steps: familiarisation by reading transcripts; finding patterns; generating concepts; coding data; sort data into categories and create themes. ${ }^{20}$ The categories and themes are described in table 2. The first author was aware of, and strove to allow for, the potential influence on the
Table 2 A summary of created categories and identified themes

\begin{tabular}{ll}
\hline Categories & Themes \\
\hline $\begin{array}{l}\text { Feel a pressure to answer questions } \\
\text { correctly }\end{array}$ & $\begin{array}{l}\text { Nervousness and } \\
\text { curiosity }\end{array}$ \\
To be insecure when performing & \\
professional skills & \\
To experience situational tension & \\
Understand own professional role & \\
Experience that own curiosity is & \\
being ignored & \\
Build relations with supervisors and & Invited and involved \\
staff & \\
Have access to information & \\
Learn as a visitor in other & \\
communities or from visitors in one's & \\
own community & \\
Learn from, with and about other & \\
professions & \\
To handle the various approaches \\
from supervisors and staff
\end{tabular}

participants. The rich combination of observations and conversation data provided possibilities to understand the culture, interactions and the participants' reflections, with an opportunity to move back and forth between the transcriptions in the emerging analysis.

The first author coded all data according to the framework, then the findings were repetitively discussed in the research group in a reflexive process, where the different perspectives and experiences contributed to build an interpretation of the data. The process continued until agreement was reached. To improve the appraisal of transferability, the setting, data collection and analysis processes were carefully described. ${ }^{22}$ Examples of field notes were presented to reinforce the analytical findings. Certain linguistic and grammatical revisions were also carried out when the field notes were translated into English. ${ }^{23}$

\section{RESULTS}

Two themes evolved characterising medical students' opportunities to participate and learn in an acute internal medicine ward. These were, Nervousness and curiosity and Invited and involved.

\section{Nervousness and curiosity}

When they first arrived at the ward, the medical students knew that they would attend it for only a short period of time. They expressed a feeling of pressure to demonstrate knowledge, to show that they were curious and that they had an interest to learn more. Students that revealed knowledge gaps during round sessions seemed to be uncomfortable and sometimes anxious, and some of them even expressed this.

The supervisors regularly put probing questions to the students. Topics could include differential diagnostic 
strategies and reasoning, pathophysiology and treatment issues and integration of evidence-based theory and practice in relation to specific patients on the ward.

At sit round sessions students were asked questions aimed to assess their level of medical knowledge. They were expected to present the patient history, medications, examination results and laboratory findings. They also had to be prepared to discuss pros and cons regarding various drug treatments and so on.

Observation of a senior consultant, two residents and one medical student. There was a calm but messy atmosphere, due to overcrowding of the ward, causing frustration among the staff. One patient was treated for convulsions caused by an earlier stroke. A discussion between the senior consultant and the medical student took place. The senior consultant put probing questions to the student. Why did we retake CRP (C-reactive protein) this morning? The student didn't know and the senior consultant told the student that convulsions could give high leukocytes and that it was important to rule out an infection. The senior consultant continued by asking which other blood tests they should be concerned with. The student could not answer and the senior consultant explained that low blood sugar levels are an aspect to be aware of in convulsion diseases.

Supervisors also requested students to explain the theoretical background of a specific test result or a physical sign in a certain patient. The supervisors' behaviour indicated that they intended to help students to integrate and use their medical theoretical knowledge in their clinical reasoning. The questioning and answering were focused solely on a specific medical problem related to a diseased organ, rather than involving the individual patient situation.

Observation of a senior consultant, a resident and a medical student. There was a calm atmosphere. One patient was treated on the ward since he had drunk cleanser, maybe because he had dementia. During the discussion between the resident and the student, the senior consultant suddenly asked: Why did he get fluid in his inferior lung lobes? The medical student correctly replied that the fluid was found there due to the structure of the lung bifurcations.

The students attended medical lectures in parallel with their placement in the ward. They were often asked questions related to the lectures. On several occasions it was the senior consultant on the ward who had given the lecture that posed the questions. Some questions were about valuing the risks and benefits of patient diagnostics and treatment, such as using a specific drug, performing a particular examination or risks associated with early discharge. Most of the questions were simple in that matter that students were supposed to provide a correct answer rather than being invited to discuss pros and cons with different solution of patient problems. Further, the questions were narrowed to specific aspects of a disease, or about diseases in a specific organ rather than more complex questions comprising the whole human being with its illness. There were only a few dialogues about how to handle a person with a complex illness, for example, concerning the patient's home situation, relationships, social networks and mental health state. Observations indicated unexplored opportunities to support students in developing competence to judge and to approach such complex patient-related problems.

Observations and conversations indicated that there were situations when the supervisors challenged the students to use practical skills that they had not previously performed, such as the insertion of a peripheral vein catheter or a urinary catheter, or to handle a tracheotomy. They pointed out to the students that it was the physician who was accountable for performing these procedures if the nurse was unable to.

Conversation between the observer and a medical student. The student expressed: "Many of these technical elements, such as inserting a urinary catheter, I had never seen performed before"

The students were repeatedly assessed individually or in groups at bedside education sessions with a supervisor. During these sessions, they had to perform tasks such as examining or proposing treatment of a patient. Uneasiness and a feeling of uncertainty could arise though the bedside education and assessment sessions differed greatly, concerning content and frequency. Students said that at some placements the sessions could take place almost everyday but at some only once a week.

Conversation between the observer and a medical student. The student pinpointed: "You do not know what you are supposed to do at each bedside education session, which is sometimes a little bit stressful but also fair because it is the same situation for every student".

The students sometimes looked tense in their interactions with supervisors. The uneasiness could arise when there were queries that the students could not answer correctly, or could increase when the senior consultant joined the team. When these situations occurred, it seemed to disturb or interrupt the learning process. There were no signs of recognition of the students' nervousness, or support of the student to manage the situation. The nervousness could be recognised by students starting to stutter, to sweat or by trembling hands.

Observation of a senior consultant, two residents and a medical student. There was a calm atmosphere. One patient was treated on the ward due to voluntary drug intoxication. A discussion between the senior consultant, the resident and the medical student took place and the senior consultant started to ask the student questions and the student started to sweat and stutter. After a while, the senior consultant had to leave the ward to take care of patients on the emergency ward. When he left the office 
the student became evidently less tense. The resident and the medical student scrutinized the electronic medical record for the last year since the patient had lost weight. When the senior consultant returned to the office the atmosphere suddenly changed and the student became uneasy again.

A transition from nervousness to curiosity could be observed when students were observed at several occasions. There were situations when the students showed an increased situational understanding of how to perform patient-related duties and to communicate with supervisors. They then could be offered the opportunity to take a more active role in the work with patients at the end of the week, and were observed to take care of patients more independently but still with support from their supervisor.

Observation of a senior consultant, a resident, a medical student, a nurse and a nurse student. There was a calm atmosphere and the resident physician summarised the patient's history. She was a young woman, being treated on the ward due to suspected pneumonia, and the circumstances were still very ambiguous. The medical student was active and curiously reading recent medical and previous medical notes. The student also made his own notes and searched for information about the disease on web sites. The student participated in a clinical reasoning dialogue with the resident about plausible differential diagnoses and what findings could confirm or reject these diagnostic hypotheses.

Some observations indicated that there were situations when the students' curiosity was ignored by the supervisors at the ward. One example was a situation when a student asked questions about a patient's psychiatric disease.

Observation of a senior consultant, two residents and a medical student. There was a calm atmosphere and patient was a woman, about 75 years old, treated on the ward due to a convulsive disorder. The student asked questions about the patient's earlier psychiatric disease history. The student didn't get any answer on the questions and the senior consultant just refers the questions to a long time of psychiatric illness.

\section{Invited and involved}

Medical students' opportunities for learning in the ward was characterised by a huge variation in conditions and possibilities to build relationships. Students were particularly involved in practice and had opportunities to perform administrative work during sit rounds and in hands-on activities. However, when staff interacted and discussed directly with patients, the students often became observers. There were situations when staff talked about the students 'above their heads' and this happened several times when the supervisor interacted with nurses or auxiliary nurses. Individual supervisors showed different levels of inclination to invite the students at work to give feedback, to stimulate them to act or to do things independently. In some circumstances, students were only able to watch while the rest of the team discussed or examined a patient or performed administrative tasks. In other cases, a student could be invited to participate as a regular team member. It was not obvious why the student was involved or not involved in the various situations. It seemed to depend mostly on whether an individual supervisor invited the student to participate or not.

Observation of a senior consultant and a medical student. There was a calm atmosphere but the senior consultant was stressed since the resident was on sick leave and the medical team lacked a member. Nevertheless, he showed a pedagogical approach to the student. He and the student moved to the observation area office. A nurse and an auxiliary nurse joined them. A male patient was treated due to convulsions. He had a long-term drug addiction and was under psychiatric custodial care. A discussion took place, initially between the senior consultant and the nurse, but the senior consultant explained to the student every aspect of what they discussed. The student asked questions about psychiatric custodial care, and both the senior consultant and the nurse explained the procedures. Then they visited the patient in the patient's room. The senior consultant presented himself and the medical student to the patient. The student examined the patient's heart and lungs on her own initiative and received feedback on her findings. The senior consultant asked the student whether she had any further questions about the patient. The student was then instructed to write the patient's discharge notes.

There were many occasions when students did not have access to information, for example, the patient's electronic healthcare records. Lack of computers hindered their possibilities to be involved in the daily work and to learn. Transferring and evaluating gathered information in relation to the patients' present state were crucial aspects of the dialogues on the ward. With only oral information it appeared that students felt that they had missed important written information hidden in inaccessible computers.

The observations showed that students' opportunities for learning occurred in and outside the ward. There were often patients to examine that had been transferred to the intensive care unit (ICU), but that remained under the responsibility of the consultant whose team the student was attached to. When at the ICU, the students had an observer role, and patient discussions took place between the expert from the ICU and the senior consultant of the ward. Some of the senior consultants introduced the students to the patient case before arriving at the ICU and summarised the case afterwards, while others did not. Students' opportunities for learning also took place at other wards in the hospital when the resident from the ward was consulted on patient-related internal medicine issues. At one occasion, a resident physician and a medical 
student visited the orthopaedic department to examine a patient. The resident introduced the student to the case beforehand. On the ward the student had the opportunity to participate in the discussion with the staff and with the patient and in the physical examination. On the way back to the ward, the resident and the medical student summarised the case together.

On a daily basis there were situations when interprofessional guidance was given on the ward. The students were invited and had the opportunity to discuss and create relationships with a variety of staff members, and not just with their own supervisor. These situations often consisted of a nurse instructing a medical student on how to, for example, take an arterial blood sample or to conduct an orthostatic blood pressure test of a patient.

\begin{abstract}
Observation of a nurse and a medical student. The observation took place in a calm atmosphere at the observation area. The student had to perform an orthostatic blood pressure examination on the patient. The nurse informed the patient and the student about how the investigation should be performed, what the student should observe and what to keep in mind: body position; time intervals, and how to check changes in blood pressure, pulse, and dizziness.
\end{abstract}

There were situations almost daily, when the students met external experts from other departments who were in the ward to examine and treat patients. In some cases, the students had the opportunity to discuss and interact with the consultants, and in other situations they were not invited at all. There were also situations when external experts guided the students in matters such as patient examinations and ways of treatment.

The dialogues indicated that the students reflected on their own personal and professional development when acting and interacting on the ward. They said that they felt more comfortable with their own learning and about their acting in various clinical situations over time during the medical programme. The students said that this progress had been obvious to them between the fifth and sixth semesters. They felt that their progress was facilitated by opportunities to be involved in the direct performance of patient care.

The students had substantial interaction with and guidance from their assigned supervisor. Most of the supervisors felt that it was stimulating and important to guide students to build their future professional competence, but the situation and organisation was not always designed to support guidance.

The dialogues indicated that short placements, some of them only one day, fragmented the learning process. Becoming a new member of a community was stressful and involved elements of social training. Students said that it was hard to get involved, to create relationships and to get a deeper understanding of the ward during short placements. The medical students emphasised enviousness of nursing students who had extended and more coherent placements.
On occasions when the atmosphere was chaotic and the workload was high, the supervisors sometimes did not have the opportunity to interact and discuss with their students. However, there were also situations when the students were not at all invited to participate in the work, even when the atmosphere seemed to be calm. Individual supervisor's approach to student involvement varied greatly, from being welcoming and friendly to ignoring them.

\begin{abstract}
Observation of a senior consultant, a resident and a medical student. There was a calm atmosphere. One female patient was being treated on the ward due to meningitis. A discussion took place between the senior consultant and the resident about the treatment of this patient. The student wasn't invited to join the discussion and was just an observer. The team took no notice at all of the student. The student seemed bored but accepted the situation without mistrust.
\end{abstract}

\section{DISCUSSION}

We have found that medical students' opportunities to participate and learn at an internal medicine ward could be characterised by the themes nervousness and curiosity and invited and involved.

\section{Nervousness and curiosity}

Stress has a dual effect on learning as described by researchers such as LePine et al. ${ }^{24}$ Hindrances and negative stress can harm the motivation to learn, and challenges can support the motivation to learn. In the present study, students sometimes felt and acted anxiously and in a stressed manner when they could not answer questions correctly, which seemed to hinder their learning process. However, we did not see any signs that this was supportive for their learning.

Students' emotional responses to clerkship have previously been described in several questionnaire and interview studies. According to Helmich et $a l,{ }^{25}$ students expressed a broad range of emotions related to the most impressive event encountered during their workplace learning. The most frequently reported negative emotions were powerless, sadness, fearfulness and nervousness. The most frequently expressed positive emotions were being moved, tenderness and respect. Further, Kasman et $a t^{26}$ has stated that insecurity, powerlessness, loss of control and a heavy workload could be negative emotional trigger pairs. Given the design of this study, we can confirm emotional responses to clerkship in acute internal medicine care by direct ethnographic observations. The students who were observed on repeated occasions over time developed curiosity, and when showing their interest, they had the opportunity to learn more.

We saw that students who were on the ward for an entire week went from a nervous to a curious state, and developed situational understanding. By the end of the week, under supervision, they could take own 
responsibility for patient care or perform a sit round by themselves. According to Wenger. ${ }^{6}$ a student can contribute to the mutual engagement in the community of practice if the members allow him or her to participate. Levitt and Cooke ${ }^{27}$ state that an extended relationship between a student and a supervisor could allow the supervisor to focus on the basics of orientation early and give the student more responsibility over time.

The learning culture on the ward was based merely on questions and answers between the medical students and different staff members. The questions covered a comprehensive area extending from simple questions that could be answered with a yes or a no to detailed and complicated medical questions on a high taxonomic level. Some questions were aimed to support integration of theoretical medical knowledge with present patient disease states. Most questions were focused on a specific medical problem related to a diseased organ rather than centred on the individual and on the whole ill human being. On rare occasions, students participated in reasoning about the life situation of the individual, and how this may affect the illness and its prognosis. However, we observed numerous situations with patients having several comorbidities, a complex social situation or addiction problems that were not addressed as learning opportunities. The supervisors seemed to focus on theoretical knowledge and medical problem solving trying to help students to come up with the right answer. A theoretical perspective of these levels of complexity in problem solving has been described by Glouberman and Zimmerman. ${ }^{28}$ Simple problems always have a precise answer. Complicated problems can be more difficult to solve. They can be broken down but are not reducible to distinct parts. Further, a complicated problem is context dependent and an understanding of the unique local conditions is mandatory. On the other hand, complex problems can include simple and complicated problems, but are not reducible to either. Complex problems often have several plausible solutions with different short-term and long-term effects, depending on how they are handled but they almost never have a single correct solution. Most of the patient cases at the studied ward include complex problems. If supervisors had approached these cases with a holistic perspective, seeing the patient as an individual with multiple diseases and socioeconomic problems, this would have given students an opportunity to develop competence to assess and approach complex patient problems.

The simple questions did not seem to increase the students' curiosity. We assume that if more detailed and complex dialogues had been initiated, new learning opportunities could have occurred. This is in line with Dyche and Epstein ${ }^{29}$ that by a relationship between the students and the supervisors, and by focusing on questions rather than answers the supervisors can challenge students to develop curiosity rather than an intention to simply produce an answer.

To sum up this theme, students sometimes acted nervous when they could not reply to questions correctly, and the nervousness could hinder their learning. Simple questions did not increase the students' curiosity. However, during a comparably short placement lasting only 1 week students showed increasing curiosity and situational understanding. We also observed potential for students to achieve learning outcomes related to judging and approaching, but these situations were not highlighted or talked about.

\section{Invited and involved}

To become involved in the community the students had to be invited by someone, for example, the supervisor, by other staff or by taking their own initiatives. We state that to be involved can facilitate development of an integrated professional embodied understanding practice and of strategies to organise medical knowledge and skills to a comprehensive professional competence as described by Dall'Alba and Sandberg ${ }^{30}$ They state that development of professional competence has two dimensions-medical expertise and embodied understanding of practice. The possibility to develop the second dimension depends on the individual students and their needs and knowledge, as well as on the learning situations.

Depending on the supervisor's attitude to the student and to supervision, the student became more or less invited and involved in the community. We observed that student opportunities to learn seemed more obvious in situations when they were accepted as members of the community and not only approached as observers. This became easier during longer placements when the students could create relationships with their supervisors. According to Wenger ${ }^{6}$, a mutual engagement appears when members are doing things together, creating relationships, when the competence of all participants is involved and all are included. It is obvious that team discussions are collaborative and patient examinations are more individual tasks, which can explain the difference in mutual engagement.

It was commonplace that students were invited to team discussions during sit round sessions but they were often excluded during patient examinations. Also Monrouxe et $a l^{31}$ have highlighted that it is rather common that supervisors exclude students from learning opportunities, as for example, patient care. Steven et $a \theta^{\theta}$ have explored that medical students learn from real patients and patient care created learning opportunities. Talk positioned the boundaries of patient care and education in three configurations: education without patient care, education within patient care and patient care without education. The nature and quality of students learning depended on if and how supervisors entered dialogue with them and linked the dialogue to authentic patient care. In this study, students often became observers when staff interacted with the patient. In contrast, students often became invited to perform patient-related paper work during, for example, sit rounds. According to McLachlan et al, ${ }^{10}$ patients may 
sometimes willingly become 'objects' from which students learn if they receive the clinical care for which they are attending a consultation and are treated respectfully. In addition, patients may become more deeply engaged in teaching consultations in which they participate actively in a triadic relationship of mutual benefit with a doctor and student. In this study, patients were positive to interact with students but we did not observe any situations where patients actually participated as teachers, giving feedback or instructions to students.

The students experienced continuous transfers between work conditions and learning situations with different possibilities to get involved. The opportunities to learn changed according to where on the ward and in what situation the students and the supervisor were. For example, students could be deeply involved in a discussion with a supervisor in the staff room but ignored by the same supervisor in his or her dialogue with a patient. The atmosphere also changed when the students visited other departments to examine a patient, and the students and the supervisors could be seen as visitors. Hagg-Martinell et al $l^{2}$ have elucidated that students' mission on arrival in a new workplace was to enter the community of practice, to understand the culture, adapt to it and try to become accepted.

Owing to the variety of conditions and the short placements, medical students had to cross community boundaries frequently. Like Wenger, ${ }^{7}$ we identified indications of exclusion, fragmentation and disconnections, but we also identified areas of extraordinary opportunities for learning, for example, when a student and a supervisor visited another ward and different perspectives intersected and new possibilities for learning arose.

As described in several other studies, for example, ${ }^{12} 32$ a good relationship with, and effective guidance from the supervisor seemed to be important for the students' learning. The supervisors on the ward did not have any scheduled time for supervision. In some cases, they created space and conditions to introduce and involve the student and in other cases did not. Maybe the lack of scheduled time for supervision in some situations created a feeling that to guide students was an extra burden to handle. This is in line with Silén $e a^{33}$ who found that allocated time for supervision allowed physicians to pay close attention to the student's learning process and to facilitate their interaction with patients.

To be invited and involved can be compared with the conclusions by Dornan et al. ${ }^{34}$ They state 'supported participation' as the core condition for workplace learning. That study was conducted with interviews before and after a 7-week clerkship period conducted in one of various healthcare settings and data were analysed with a grounded theory approach. Our findings show that similar conditions apply even during short placements in an acute internal medicine care ward.

There were no indications that the staff expressed curiosity about the students as individuals or if the students had any ideas about things or routines to improve at the ward. Only a few discussions were about the students' earlier educational experiences or about their expectations and future plans. The lack of curiosity from the staff in these situations could be explained, according to Wenger ${ }^{6}$ as indicating that students are legitimate but peripheral members of the community.

Given the design of this study, we could not identify any obvious development or learning of the organisation or the routines due to the influence of student participation. We could speculate that the high turnover of students and staff on the ward could be a hindrance to such a development. According to Wenger ${ }^{7}$, newcomers such as students can develop a community if they participate and get involved. They can act like brokers, using their earlier experiences from different places and cultures, and from their interactions with members in other communities of practice. A broker is someone who can introduce elements of one practice to another. However, brokers often do not fully belong anywhere and may not add directly to any specific result, the evaluations they bring can easily be ignored.

In most of the learning situations observed there were excellent potentials for students to achieve their intended learning outcomes. However, the community of practice culture in the ward, more thoroughly described in our previous publication, ${ }^{19}$ were potential hindrances for learning. The interrogations and the pressure to know and to show interest in learning could be seen as hindrances and as facilitators of learning. Several of the students had just short 1 day placements. Those staying for 1 week or more were observed to establish relationships with supervisor and to become involved in the community. The students also valued being in a team and having the opportunity to create relationships with, and to learn from, staff members of differing professions.

To sum up this theme, short placements are potential hindrances for opportunities to learn. It took several days for the students to create relationships to their supervisor, and to get invited and involved in the community. Shorter placements therefore also make it difficult for the community to learn from the students and for the healthcare organisation to take advantage of the students' experiences.

\section{METHOD DISCUSSION}

Qualitative studies are performed in a specific context and create an opportunity for a deeper analysis of the studied context. The ethnographic design provided a potential to gain a deeper understanding of participants' opinions and actions, and the extended observation period of a large number of staff and student interactions increased the findings' trustworthiness and credibility. A limitation of ethnographic studies is a risk of bias of the researcher, as the researcher is used as a tool during the observations. The observer (the first 
author) was a female registered nurse with a long experience of nurse education in acute internal medicine care settings. This preunderstanding and experience of the context allowed the observations and the analysis to be performed more in depth. In contrast, a researcher with preunderstanding and experience of a studied context may contribute to that important aspects are not noticed. None of the observed individuals reacted in a negative way during the observations. Initially, some of the staff were a bit cautious, but they also expressed that the research question was important to investigate and that they wanted to contribute. By using 'marginal participant' technique, in which the researcher plays only a minimal role in the interaction, as we have done in this study, this risk is decreased. The observer took a nonparticipating role during the observations to focus on observing what happened in the room, how the participants were placed and what interactions that took place. Furthermore, investigator triangulation was applied and all researchers were engaged in the analysis, helping each other to identify preconceptions and biases that might colour their interpretation. By applying different professional perspectives and experiences, credibility could be ensured. The research group consisted of a nurse (the first author), two senior consultants and a senior educational researcher. All four had long experiences of education, mainly in acute hospital settings. Gathering of observation information during an extended time and information from informal conversations with staff and students further ensured credibility. We have studied medical students during early clerkship at an acute internal medicine care ward and the results might be transferable to early clerkship in similar healthcare settings.

\section{CONCLUSION}

This study shed light on how an acute internal medicine ward culture can facilitate medical students' possibilities to participate and learn. Medical students' learning situations in this context was focused on questions and answers rather than dialogues related to the whole ill human being. Students had to put up with continuous transfers between variable learning conditions and learning situations where the potential to be involved varied. These results can be useful in development of workplace learning in acute care, for example, by creating longer placements, and by encouraging the potential to challenge students in dialogues exploring the complexity of presented patient cases.

Acknowledgements The authors thank all the participants for giving their time to take part in this study.

Contributors AK took initiative to the study and $\mathrm{HH}$ came up with the idea to apply an ethnographic design. AHM performed, under supervision of AK, all observations and acquisition of data and compiled the written documentation of the observational data. All four authors (AHM, HH, PH and AK) contributed substantially to analysis and interpretation of data for the work. AK and AHM performed the main part of drafting the work and then all authors (AHM, $\mathrm{HH}$, $\mathrm{PH}$ and $\mathrm{AK}$ ) contributed substantially in revising it critically for important intellectual content. All four authors (AHM, HH, PH and AK) have approved the final submitted version and all four authors (AHM, HH, PH and AK) have agreed to be accountable for all aspects of the work in ensuring that questions related to the accuracy or integrity of any part of the work are appropriately investigated and resolved.

Funding The study was supported by grants provided by the Stockholm County Council (ALF project).

Competing interests None declared.

Ethics approval Regional Ethical Review Board, Stockholm Sweden no 2011/ $1268-31 / 5$

Provenance and peer review Not commissioned; externally peer reviewed.

Data sharing statement No additional data are available.

Open Access This is an Open Access article distributed in accordance with the Creative Commons Attribution Non Commercial (CC BY-NC 4.0) license, which permits others to distribute, remix, adapt, build upon this work noncommercially, and license their derivative works on different terms, provided the original work is properly cited and the use is non-commercial. See: http:// creativecommons.org/licenses/by-nc/4.0/

\section{REFERENCES}

1. Dornan T. Workplace learning. Perspect Med Educ 2012;1:15-23.

2. Irby DM. Clinical teaching and the clinical teacher. J Med Educ 1986;61(Pt 2):35-45.

3. Aper L, Reniers J, Derese A, et al. Managing the complexity of doing it all: an exploratory study on students' experiences when trained stepwise in conducting consultations. Bmc Med Educ 2014;14:206.

4. Mennin S. Self-organisation, integration and curriculum in the complex world of medical education. Med Educ 2010;44:20-30.

5. Vygotskij L. The collected works of L.S. Vygotsky. The history of the development of higher mental functions. Vol 4. New York: Plenum Press, 1997.

6. Wenger E. Communities of practice: learning, meaning, and identity. Cambridge: Cambridge University Press, 1998.

7. Wenger E. Communities of practice and social learning systems. Organization 2000;7:225-46.

8. Wenger-Trayner E, Wenger-Trayner, B. Learning in landscapes of practice: boundaries, identity, and knowledgeability in practice-based learning. Routledge, 2014.

9. Steven $\mathrm{K}$, Wenger $\mathrm{E}$, Boshuizen $\mathrm{H}$, et al. How clerkship students learn from real patients in practice settings. Acad Med 2014;89:469-76.

10. McLachlan E, King N, Wenger E, et al. Phenomenological analysis of patient experiences of medical student teaching encounters. Med Educ 2012;46:963-73.

11. Karani R, Fromme HB, Cayea D, et al. How medical students learn from residents in the workplace: a qualitative study. Acad Med 2014;89:490-6.

12. Hägg-Martinell $\mathrm{A}$, Hult $\mathrm{H}$, Henriksson $\mathrm{P}$, et al. Students perceive healthcare as a valuable learning environment when accepted as a part of the workplace community. Educ Health (Abingdon) 2014:27:15-23.

13. Smith SE, Tallentire VR, Cameron HS, et al. The effects of contributing to patient care on medical students' workplace learning. Med Educ 2013;47:1184-96.

14. Strand $P$, Edgren $G$, Borna $P$, et al. Conceptions of how a learning or teaching curriculum, workplace culture and agency of individuals shape medical student learning and supervisory practices in the clinical workplace. Adv Health Sci Educ Theory Pract 2015;20:531-57.

15. Liljedahl M, Boman LE, Fält CP, et al. What students really learn: contrasting medical and nursing students' experiences of the clinical learning environment. Adv Health Sci Educ Theory Pract 2015;20:765-79.

16. Atherley $\mathrm{AE}$, Hambleton IR, Unwin N, et al. Exploring the transition of undergraduate medical students into a clinical clerkship using organizational socialization theory. Perspect Med Educ 2016:5:78-87.

17. Köhl-Hackert N, Krautter M, Andreesen S, et al. Workplace learning: an analysis of students' expectations of learning on the ward in the Department of Internal Medicine. GMS Z Med Ausbild 2014;31: Doc43.

18. Seabrook MA. Clinical students' initial reports of the educational climate in a single medical school. Med Educ 2004;38:659-69. 
19. Hägg-Martinell $A$, Hult $H$, Henriksson $P$, et al. Community of practice and student interaction at an acute medical ward: an ethnographic study. Med Teach 2016;38:793-800.

20. Hammersley M, Atkinson P. Ethnography: principles in practice. 3rd edn. London: Routledge; 2007.

21. Atkinson P. The clinical experience: the construction and reconstruction of medical reality. 2nd edn. Ashgate: Aldershot, 1997.

22. Patton M. Qualitative research \& evaluation methods. London: SAGE, 2002.

23. Kvale S, Brinkmann S. Transcribing interviews. In InterViews: learning the craft of qualitative research interviewing. 2nd edn. Los Angeles: Sage Publications, 2009.

24. LePine JA, LePine MA, Jackson CL. Challenge and hindrance stress: relationships with exhaustion, motivation to learn, and learning performance. J Appl Psychol 2004:89:883-91.

25. Helmich E, Bolhuis S, Prins J, et al. Emotional learning of undergraduate medical students in an early nursing attachment in a hospital or nursing home. Med Teach 2011;33:e593-601.

26. Kasman DL, Fryer-Edwards K, Braddock CH, III. Educating for professionalism: trainees' emotional experiences on IM and pediatrics inpatient wards. Acad Med 2003;78:730-41.
27. Levitt DS, Cooke M. Tips for teaching in longitudinal clerkships Clin Teach 2011;8:93-6.

28. Glouberman S, Zimmerman B. Complicated and complex systems: what would sucesssful reform of medicare look like? Toronto: University of Toronto Press Inc., 2004.

29. Dyche L, Epstein RM. Curiosity and medical education. Med Educ 2011:45:663-8.

30. Dall'Alba G, Sandberg J. Unveiling professional development: a critical review of stage models. Rev Educ Res 2006;76:383-412.

31. Monrouxe LV, Rees CE, Dennis I, et al. Professionalism dilemmas, moral distress and the healthcare student: insights from two online UK-wide questionnaire studies. BMJ Open 2015;5:e007518.

32. Stenfors-Hayes T, Hult H, Dahlgren L. What does it mean to be a good teacher and clinical supervisor in medical education? Adv Health Sci Educ 2011;16:197-210.

33. Silén C, Kiessling A, Spaak J, et al. The experience of physician supervisors with clerkship students: a qualitative study. Int $J$ Med Educ 201156-63.

34. Dornan $\mathrm{T}$, Boshuizen $\mathrm{H}$, King $\mathrm{N}$, et al. Experience-based learning a model linking the processes and outcomes of medical students workplace learning. Med Educ 2007;41:84-91. 